\title{
Philosophiques
}

\section{Merleau-Ponty et le marxisme ou La difficulté de tolérer l'intolérable}

\section{Jean-Luc Gouin}

Volume 18, numéro 1, printemps 1991

URI : https://id.erudit.org/iderudit/027142ar

DOI : https://doi.org/10.7202/027142ar

Aller au sommaire du numéro

Éditeur(s)

Société de philosophie du Québec

ISSN

0316-2923 (imprimé)

1492-1391 (numérique)

Découvrir la revue

Citer cet article

Gouin, J.-L. (1991). Merleau-Ponty et le marxisme ou La difficulté de tolérer l'intolérable. Philosophiques, 18(1), 95-117. https://doi.org/10.7202/027142ar
Résumé de l'article

L'extrême barbarie de la seconde guerre mondiale, et l'espoir d'une vie différente pour l'humanité, ont amené M. Merleau-Ponty à réfléchir sur le marxisme - théorique d'une part, stalinien d'autre part. Notre projet consiste à suivre son cheminement intellectuel à cet égard depuis ses premiers écrits politiques de '45 jusqu'en I960, quelques mois à peine avant sa mort subite survenue il y a exactement trente ans, en mai 1961. Mais en quoi est-il pertinent, à l'heure où un vent d'Histoire plane sur l'Est, de revenir sur des textes dont certains atteindront bientôt le demi-siècle ? C'est que l'auteur a vu clair avant presque tout le monde, de telle sorte qu'il réussit à identifier, inscrite dès l'origine dans son propre système, l'asphyxie inévitable du régime soviétique. Aussi, lire le Merleau-Ponty de ces années pas si lointaines, c'est lire à notre avis l'URSS de Gorbatchev et voir également s'épanouir la nouvelle Europe depuis Prague jusqu'à Sofia. C'est comprendre du même souffle - ce que n'eût point désavoué Marx - qu'on ne saurait bâillonner indéfiniment, enracinée dans le tissu de l'homme, la Volonté-de-liberté des peuples et des nations.
Ce document est protégé par la loi sur le droit d'auteur. L'utilisation des services d'Érudit (y compris la reproduction) est assujettie à sa politique d'utilisation que vous pouvez consulter en ligne.

https://apropos.erudit.org/fr/usagers/politique-dutilisation/ 
PHILOSOPHIQUes, Vol. XVIII, Numéro1, Printemps 1991

\title{
MERLEAU-PONTY ET LE MARXISME OU LA DIFFICULTÉ DE TOLÉRER L'INTOLÉRABLE
}

\author{
par Jean-Luc Gouin
}

Ces décisions qui tranchent sont pour moi ambiguës.

Le visible et l'invisible

RÉSUMÉ. L'extrême barbarie de la seconde guerre mondiale, et l'espoir d'une vie différente pour l'humanité, ont amené M. Merleau-Ponty à réfléchir sur le marxisme - théorique d'une part, stalinien d'autre part. Notre projet consiste à suivre son cheminement intellectuel à cet égard depuis ses premiers écrits politiques de ' 45 jusqu'en 1960, quelques mois à peine avant sa mort subite survenue il y a exactement trente ans, en mai 1961. Mais en quoi est-il pertinent, à l'heure où un vent d'Histoire plane sur l'Est, de revenir sur des textes dont certains atteindront bientôt le demi-siècle ? C'est que l'auteur a vu clair avant presque tout le monde, de telle sorte qu'il réussit à identifier, inscrite dès l'origine dans son propre système, l'asphyxie inévitable du régime soviétique. Aussi, lire le MerleauPonty de ces années pas si lointaines, c'est lire à notre avis I'URSS de Gorbatchev et voir également s'épanouir la nouvelle Europe depuis Prague jusqu'à Sofia. C'est comprendre du même souffle - ce que n'eût point désavoué Marx - qu'on ne saurait bâillonner indéfiniment, enracinée dans le tissu de l'homme, la Volonté-de-liberté des peuples et des nations.

ABSTRACT. The extreme barbarity of the Second World War, and the hope for a different life for Humanity, lead Merleau-Ponty to reflect on marxism, theoretical as well as Stalinian. Our aim is to follow his intellectual progress on this subject since his first political writing, from 1945 until 1960, that is, just a few months before his sudden death in may 1961. However, where is the relevance to come back to these fifty years old texts when History blows on eastern countries? Simply because Merleau- 
Ponty has foreseen the present situation almost before any other analyst, in such a way that he identified successfully the unavoidable asphyxia of the soviet regimen, as inscribed at the very outset of this political system. To read the Merleau-Ponty of these years, it is, do we think, to read the USSR of Gorbatchev, and to sec also the new Europa to expand, from Prague to Sofia. It is also to understand, by the same occasion - and Marx would not have disavowed it -, that one cannot silence indefinitely the Will of liberty of people and nations which is routed in the nature of man. 


\section{5-1947 : De l'Humanisme à la Terreur}

L'au teur approchait déjà la quarantaine lorsqu'il éprouva l'urgence de réfléchir sur les événements politiques. Son itinéraire phénoménologique dans la lignée de Husserl ne le prédestinait pas particulièrement à ce champ nouveau sinon peut-être, et très indirectement, par la cinquième des Méditations cartésiennes (1931) et la Krisis (dont le texte en entier ne devint toutefois disponible qu'en 1954). C'est dire que les événements crus comme le froid de Sibérie l'ont éveillé à la tragédie humaine, pour laquelle souffrir et mourir est plus aisé encore que penser librement au bord de la Seine. ${ }^{1}$

Il y eut tout d'abord la guerre, tout aussi réelle qu'impossible : je suis de cette génération, dit l'auteur, qui croyait que "la guerre de Troie pouvait encore n'avoir pas lieu *. ${ }^{2}$ Elle est passée de réelle à infernale sous l'occupation. C'était un dur coup porté à la transparence intelligible du cogito issu de Descartes, devenu donateur de sens sous la plume de Husserl: alors que tant d'hommes ne jouissent pas de l'été qui accueille la Libération, qui donc maintenant donnera le sens? Décidément, il fallait se demander si la mise entre parenthèses (ou épocbè) n'était pas au fond une mise en boîte.

À la recherche d'une existence moins désespérante, le philosophe se tourne vers la pensée marxiste et son rejeton soviétique. Symétriquement, il pose un œil sévère sur le libéralisme occidental pour lequel la violence est * supprimée dans le commerce des idées, mais maintenue dans la vie effective, sous la forme de la colonisation, du chômage et du salaire. ${ }^{3}$ Nos nobles idéaux de raison et de liberté sont devenus ces manteaux d'Arlequin que même le climat africain - algérien notamment - supporte avec grand peine. Et la société se révèle d'autant plus répugnante qu'elle se réclame avec ostentation de ces principes qui

1. Maurice Merleau-Ponty (1908-1961) est surtout connu, en effet, dans la mouvance du grand courant phénoménologique contemporain, et notamment pour ses thèses d'État sur la Structure du comportement et la Pbénoménologie de la perception, publiées respectivement en 1942 et 1945. Il fut également co-fondateur et, jusqu'en 1953, codirecteur avec Jean-Paul Sartre de la revue Les Temps Modernes.

2. Sens, p. 246 . Voir le code des abréviations en fin de texte

3. Humanisme, p. 205. Ce livre est né sous l'impulsion du roman d'Arthur Koestler, Le Zéro et l'infini (publié à Paris en 1945), dénonçant le totalitarisme soviétique. L'ouvrage fit réfléchir M.-P. qui l'avait déjà lu dans sa version originale, parue sous le titre Darkness at noon en 1940. Humanisme et terreur lançait en 1947 le débat sur la place publique française. 
farcissent ses hymnes et ses discours. En somme, il faut voir qu'«un régime nominalement libéral peut être réellement oppressif. " ${ }^{4}$ L'auteur s'adresse alors à l'entreprise de Marx parce qu'elle demeure, dit-il, «le seul humanisme qui ose développer ses conséquences. ${ }^{5}$ Il endosse le projet au point d'affirmer que «ce n'est pas une philosophie de l'histoire, c'est la philosophie de l'histoire, et y renoncer, c'est faire une croix sur la Raison bistorique. " 6

M.-Ponty se montre visiblement enthousiasmé par son premier contact avec cette pensée aux mille promesses. Il y trouve une soif authentique de liberté pour tous les hommes, certes. Mais plus encore, il y découvre cette volonté de se dégluer du moralisme (spécieux par son inactivisme chronique) en vertu de son acharnement à débusquer de la réalité historique - concrète - les visages de l'aliénation. Ce qui a comme effet d'engendrer la condition même de leur dépassement vers une société plus juste, voire idéale.

Par ailleurs, à cette époque, l'auteur appréhende le politique avec les instruments phénoménologiques (gonflés, on le sait, de sens et donc d'espoir) qui ont eu leur efficace dans les grandes thèses qu'il venait de publier. Pour lui, en effet, être marxiste "c'est penser que l'histoire est une Gestalt ", concept qu'il emprunte à Koehler, et c'est considérer que * tout est vrai à sa place et à son rang dans le système total de l'histoire, tout a un sens. ${ }^{7} \mathrm{Au}$ coeur de l'histoire que constitue le prolétariat s'entremêle en outre "l'intersubjectivité humaine concrète" - la révolution sociale rencontre celle du sujet phénoménologique. Voilà qui encourage l'ouverture et, pourquoi pas, la bienveillance envers la tentative soviétique. Après tout, "les Russes, cela signifie un minimum de politique prolétarienne.. 8

Pourtant si le même homme se définit clairement, sinon rigoureusement, comme un sympathisant à la cause "communiste", il se refuse à toute adhésion. On reconnaît ici la lucidité du philosophe. Non pas que celui-ci reste interdit devant l'éventualité de l'agir. Mais lorsque cette action lui paraît en quelque façon ir-(ou a-)rationnelle, il ne peut $y$ consentir qu'avec grande prudence, et certes pas avant d'y avoir réfléchi sérieusement. De plus, dans l'esprit du com-

4. Humanisme, p. 41 .

5. Humanisme, p. 270

6. Humanisme, p. 266. Nous soulignons.

7. Humanisme, p. 237 et Sens, pp. 226-227

8. Humanisme, p. 274. 
mentateur, outre la mollesse de la pensée, l'irrationalité signifie fréquemment violence parce qu'elle l'implique si aisément (comme le soulignait déjà avec pertinence Eric Weil à la même époque). Humanisme et terreur, publié au lendemain de la guerre, s'annonce précisément comme une réflexion - tourmentée - sur la légitimité de la violence dans un projet dont "Liberté " est le maître mot. Dès les premières lignes de l'ouvrage nous sommes fixés : du communisme, il s'agit de savoir «si la violence qu'il exerce est révolutionnaire. ${ }^{9}$ Car en politique, ne pas craindre les mots ne suffit pas. On doit se rendre à l'évidence que les symétries faussent le débat : "Nous n'avons pas le choix entre la pureté et la violence, mais entre différentes sortes de violence. "10

Déjà transparaît l'ambiguité de la réflexion merleaupontyenne, consécutive à la difficulté de la tâche qui est celle d'interpréter les événements. Car si le marxisme demeure, comme Sartre le dira plus tard, l'indépassable philosophie de notre temps, et que s'il est illusoire de penser procéder à son accouchement sans douleur aucune, il devient rien moins qu'aberrant de laisser l'enfant à lui-même, sans examen. Aussi est-on constamment renvoyé, dans Humanisme, du * oui » au * mais». Oui à la liberté, oui à une vie d'homme qui refuse sa propre réification: oui, mais pas à n'importe quel prix! Or le sens que livre la situation soviétique n'a pas la clarté de la géométrie analytique. C'est pourquoi l'adhésion y côtoie le refus moins par inconstance du jugement que par l'absence de paramètres solides, permettant de départager le tolérable de l'inacceptable. Du reste le sens réside peut-être, nous dit l'auteur, dans le résultat social à-venir, et échapperait de la sorte à la lunette du maintenant.

Aussi voit-on notre analyste déchiré entre ses espoirs marxistes et les opacités du réel communiste ${ }^{11}$ qu'il s'emploie parfois à justifier sans réussir à se convaincre totalement lui-même. D'où le flottement de la pensée. Le philosophe préfèrerait dire sans doute, à ce sujet, que les aléas de l'histoire ne peuvent à ce jour être évalués de façon définitive. Il constate que le système fondé en principe sur le prolétariat lui accorde maintenant bien peu de place, et

9. Humanisme, p. 44.

10. Humanisme, p. 213

11. C'est ainsi que M.-P. qualifiait le régime stalinien bien que le communisme soit, au sens strict, comme on le sait, le système politique dans lequel l'abolition de l'État rejoint la disparition des classes réalisée progressivement, en principe, depuis l'étape du socialisme. Le régime sovietique correspondait theorique. ment au socialisme d'État, que d'aucuns nommèrent parfois avec humeur capitalisme d'État. 
que celui-ci «a cessé d'être le terme de référence de la pensée communiste. ${ }^{12}$ Merleau-Ponty n'ignore pas non plus que la politique soviétique d'industrialisation et de collectivisation forcée ne se contente point de casser quelques œufs pour les besoins du repas collectif, ni d'ailleurs qu'un système de privilèges aux membres de la Nomenklatura renvoie les espoirs de 1917 aux calendes pré-romaines. La reconnaissance tant attendue de l'homme par l'homme se meut et se meurt donc en des sentiers obscurs et peu accueillants. Le dépérissement de l'État? Allez donc en parler au Comité central! Par delà les événements, même la philosophie (de/du Parti) pointe ses armes : «La dialectique est en fait remplacée par le rationalisme scientiste. ${ }^{13}$ D'autre part, quiconque critique ou interroge les agissements des nouveaux maîtres essuie aussitôt la salissante épithète de "réactionnaire» - encore heureux de parvenir, le cas échéant, à esquiver celle de traître. Car «il leur faut des coupables tout noirs, des innocents tout blancs. ${ }^{14}$

Le chemin de croix que constitue Humanisme et terreur pour celui qui le rédigea eut comme effet, selon nous, de faire prendre conscience à l'auteur que marxisme et communisme ne sont pas, comme il l'espérait, les termes historico-pratiques répondant docilement aux notions de théorie et de praxis. L'intellectuel français se voit finalement médusé par la fissure béante qu'il découvre entre le rigoureux penseur de la révolution et cette *sorte de néo-communisme assez voisin du pragmatisme. " 15

De ce commentaire qui ne cache point son indignation, faut-il conclure que le philosophe évacue formellement le marxisme de notre temps? Merleau-Ponty écrira notamment qu'il est possible «de composer un montage qui représente la vie soviétique à l'opposé de l'humanisme prolétarien. ${ }^{16}$ Or voilà à notre avis un propos qui illustre comme en raccourci le dilemme du livre: les mêmes expressions forment antithèse avec elles-mêmes. L'auteur ne sait pas encore vraiment si le communisme dessiné par ce montage est une construction théorique erronée (c'est-à-dire un constat établi en vertu d'une mauvaise lecture des événements), ou celui de l'histoire bien réelle dégénérant en tragédie. Ses

12. Humanisme, p. 252.

13. Humanisme, p. 47. Thèse qui sera par ailleurs amplement developpée dans ses Aventures.

14. Humanisme, p. 67 .

15. Humanisme, p. 255.

16. Humanisme, p. 245. 
espoirs lézardés s'accrochent pourtant au "sens" prochain encore possible, à savoir le retour au marxisme autbentique, qui permettrait un virement de barre de la politique à la Staline versant *dans l'idolâtrie de l'objectivité. * ${ }^{17}$ En dernière analyse, M.-Ponty estime que la rélexion de Marx "reste possible non seulement à titre de critique morale, mais même à titre d'hypothèse historique. " 18

\section{De l'Histoire à la Philosophie}

Huit années séparent Les Aventures de la dialectique 19 de Humanisme et terreur. Ces années furent déterminantes pour la pensée de Merleau-Ponty. Elles n'ont pas manqué du reste, sur le plan politique, de se révéler importantes également un peu partout autour du globe. Pour les événements, il faut noter plus spécialement la fiévreuse activité de l'URSS. Pendant qu'elle participe à la guerre de Corée et envoie ses chars à Berlin (en 1953), elle ne peut empêcher l'Occident de prendre une conscience plus aiguë que jamais de l'existence des "camps de travail", dont l'euphémisme n'en rend la réalité que plus méprisable. La guerre de Corée révélera de façon très nette, aux yeux de l'auteur, combien le déploiement de la puissance de l'Est ne rejoint aucunement la lutte des prolétariats locaux et qu'elle n'est, au contraire, que la conséquence militaire de visées impérialistes. Les camps, quant à eux, dévoileront le fieffé mensonge que constitue un régime qui fonde sa *démocratie populaire" sur la répression systématique.

C'en est trop! «Le communisme pratique le choix injustifiable. ${ }^{20}$ La contradiction entre le marxisme et la société qui s'en réclame amène notre philosophe à percer le théâtre du geste pour étudier le scénario dont il s'inspire. S'aidant du sociologue Weber, il décide donc d'interroger la *Sainte famille" nouvelle version en rencontrant les Lénine, Trotsky, Lukacs ainsi que le Sartre frais émoulu des * Communistes et la paix»: «Il doit y avoir dans la critique même, pense-t-il, quelque chose qui prépare les défauts de l'action. ${ }^{21}$

Et de fait, M.-Ponty découvre dans le texte l'assommoir qui opprimera la parole vivante. Il s'agit à ses yeux de

17. Sens, p. 182.

18. Humanisme, p. 270

19. Publié à Paris chez Gallimard, en 1955

20. Aventures, p. 250

21. Aventures, p. 338 
l'insidieuse métamorphose de la théorie (c'est-à-dire l'essai rigoureux de compréhension d'un secteur du réel) en une dogmatique qui, par définition, prétend de façon absolue atteindre le vrai et qui, dès lors, repousse comme vaine et malvenue toute critique portant sur ses énoncés. Plus spécifiquement, il faut reconnaître dans la pensée marxiste la transposition de concepts opératoires en réalités proprement ontologiques : les instruments du penser deviennent des "choses" dans le monde. Ainsi en est-il notamment du dialectique. Le processus se déploie également par le truchement de réalités concrètes et aisément identifiables, tel le prolétariat, lequel se voit assumer les promesses venues d'ailleurs qu'on persiste à lui insuffler de façon présumément objective.

En 1908, Lénine publie Matérialisme et empiriocriticisme. Malgré le respect que notre auteur porte à ce génie du siècle naissant, plus que tout autre ce texte devait s'avérer le boulet au pied que de nombreux partisans, cependant, reçurent comme la baïonnette agile au bout de laquelle devait s'épanouir la rose du meilleur avenir. Marx avait déjà montré avec force combien les rapports entre les hommes sont médiatisés par les choses. On a compris depuis lors que les rapports de production constituent le lieu privilégié où l'économique se fait politique, c'est-à-dire où se déroule la lutte des pouvoirs et s'instaure la réification de l'homme sous le couvert de la « libre*-concurrence. Mais l'auteur du Capital ne s'arrête pas là. Il estime que ce réel bigarré et trompeur recèle en soi la logique de son propre dépassement. C'est dire qu'une lecture lucide de l'histoire et de la société capitaliste nous amènerait à considérer que cette société périra - dialectiquement - de ses propres contradictions: la révolution gronde dans l'aliénation du plus grand nombre et le pouvoir doit échoir, selon l'expression de Sartre, à "ceux qui ne sont rien."

Or il s'agit maintenant de savoir comment lire Marx dans-le-texte. L'étude de Lénine ne s'éternise pas outre mesure sur les nuances dans le style de Weber - lequel suggère en outre de distinguer le sens qu'on découvre dans les événements du concept de force auquel il est fréquemment associé. Et pourtant, dira M.-Ponty, tout le problème du marxisme réside sans doute dans cette question qu'on estime résolue en la qualifiant d'«abstraite* ou, mieux, de bourgeoise. Mais lorsque Marx en appelle à la dialectique du réel économique pour promouvoir l'élan révolutionnaire du prolétariat, se réfère-t-il à des lois scientifiques, à 
un prophétisme délirant, ou nous sert-il quelque concoction bizarre où l'esprit rigoureux se mêle à une soif par ailleurs passionnée de liberté pour tout ce qui est humain?

Il semble assez clair pour notre philosophe que le matérialisme léninien phagocyta la dialectique de façon expéditive. Si celle-ci, en effet, est inscrite dans les choses à la manière du sceau sur le document, il appert que nous avons là une dialectique dont on chercherait en vain le «mouvement». Hier, regard stroboscopique sur la société des hommes, elle devient maintenant pareille aux multiples pétrifications qu'elle avait jadis précisément permis d'identifier derrière le rideau sauvage de l'économie. Ce fameux concept n'est plus le propre de la science, ni même un outil euristique, moins encore structure du penser. Il fait partie du monde comme le mobilier de la salle à manger. Il est devenu chose parmi les choses.

La litigieuse théorie du reflet constitue la plaque tournante qui permit au penseur de la Révolution russe d'aménager son * nouveau * marxisme. En substance, Lénine pose que la conscience réifiée - parce que réifiée - ne saurait atteindre la vérité, c'est-à-dire la compréhension du milieu dans lequel elle baigne. ${ }^{22}$ Conscience et monde (ou sujet et objet) sont ainsi renvoyés dos à dos dans un rapport à donner des sueurs au Sphinx de Thèbes. Si la vérité échappe à la conscience, en effet, d'où vient alors la connaissance digne de ce nom, et par qui ou quoi vient-elle au jour? Transis par l'aliénation, nous ne pouvons comprendre cette réalité, soit. Reste cependant, et ici M.-Ponty paraphrase Lénine, que nous pouvons «l'expliquer par le processus objectif de l'économie. ${ }^{23}$ Dès lors, l'individu qui désire parvenir à y voir clair, et le cas échéant manifester son emprise sur la réalité sociale, n'a d'autre choix que l'emprunt d'une voie unique : la rationalité sans sujet. C'est ce qu'on pourrait appeler l'objectivisme dans l'uniforme du matérialisme.

Il y a donc une vérité des choses qui tout en nous échappant nous contraint. C'est exactement de la magie, constate notre critique. Et on comprendra que celui-ci parle du mariage de «la dialectique avec la métaphysique matérialiste $(.$.$) ce mélange instable de hégélianisme et de scien-$

22. On se souviendra de la conscience faussee (aliénée) dont nous parle Marx, fruit amer d'un monde d'hommes caché sous les choses et, par surcroír, copieusement nourrie de l'idéologie * dominante *

23. Aventures, p. 96 . Nous soulignons. 
tisme." La théorie, poursuit-il, "rend à la dialectique un fondement absolu dans l'être ou dans l'objet pur et revient ainsi, non seulement en deçà du jeune Marx, mais en deçà de Hegel. ${ }^{24}$ Ce que l'homme de la praxis laisse dans l'ombre, et que Lukacs voudrait lui rappeler en 1923,25 c'est que le savoir et la vérité sont toujours le résultat du travail d'un sujet ou, si l'on préfère: l'objectivité demeure inévitablement l'objectivité d'une subjectivité. Lénine choisit de croire que les choses parlent d'elles-mêmes. Notre auteur a magnifiquement cerné la difficulté en un langage dont la concision justifie la longue appropriation que nous faisons de son texte :

À partir du moment en effet où la conscience et l'être sont posés face à face comme deux réalités extérieures, où la conscience, comme simple reflet, est frappée d'un doute radical, et l'histoire, comme seconde nature, affectée d'une opacité qui ne peut jamais être entièrement réduite, la conscience ne dispose plus d'aucun critère pour distinguer en elle-même ce qui est connaissance et ce qui est idéologie, et le réalisme naiff, comme il l'a toujours fait, se conclut au scepticisme. S'il échappe à cette conséquence, ce ne peut être que par un coup de force, par une adhésion immotivée à quelque instance extérieure - le processus social en soi, le Parti - et toutes les productions de la pensée désormais devront être mesurées à cet étalon. ${ }^{26}$

Par delà Lénine, ici s'exprime l'essentiel de la critique merleau-pontyenne des épigones de Marx: en devenant chose la vérité perd son nom, et devient l'objet d'un culte et l'alibi de toutes les croisades. Aussi n'est-il pas incongru de rappeler que

beaucoup de marxistes (...) ne voient pas que si la conscience était jamais absolument coupée du vrai, ils seraient euxmêmes réduits au silence et que nulle pensée, pas même le marxisme, ne pourrait prétendre à la vérité. ${ }^{27}$

Il faut dire que déjà chez Engels et Plékhanov on assiste, pense le commentateur, à une naturalisation de la dialectique. D'ailleurs de ces deux marxistes, dit-il, «on passe facilement aux vues de l'orthodoxie contemporaine. 28 Trotsky n'y échappe point. Son concept de "Révolution permanente» ne va pas sans charmer tout d'abord notre philo-

24. Aventures, p. 99

25. Anné de la parution à Berlin (bien que l'auteur soit hongrois) d'Histoire et conscience de classe, livre maudit par l'orthodoxie marxiste.

26. Aventures, p. 101 . Nous soulignons.

27. Aventures, p. 64

28. C'est-à-dire au positivisme systématique. Aventures, p. 96. 
sophe qui toutefois, après mûre réflexion, se rend à l'évidence que * pour s'installer sur le terrain de l'histoire, il faut qu'ils [le Parti et le gouvernement, même révolutionnaires] existent positivement ${ }^{29}$ ". Bien que d'un matérialisme moins plat, l'auteur de La Révolution trabie demeure quand même tout aussi objectiviste, et par conséquent contradictoire. De fait, son texte exprime ouvertement «la conviction philosophique que la société homogène et sans État est virtuellement donnée avec la fin du capitalisme, que cette dialectique est dans les choses * - voilà qui permet de "donner au pouvoir prolétarien cette investiture philosophique. " 30

Or s'il est écrit, il n'est pas établi pour autant que la fin du capitalisme signifie de facto le pouvoir du prolétariat et, enfin, la société sans classes. ${ }^{31}$ Il est certes rassurant de songer que la solution à nos problèmes veille quelque part dans le monde, et qu'il suffit d'apprivoiser l'objet dans le filet de nos espérances pour le transformer en fer de lance vers des lendemains qui chantent. Néanmoins, depuis 1917 il n'a pas été prouvé qu'un jour, forcément, «un pouvoir prolétarien sera "mûr", ni qu'un pouvoir révolutionnaire soit jamais autrement que "prématuré". . ${ }^{32}$ Il est légitime de s'interroger sérieusement. Le concept de prolétariat ne serait-il pas au fond un porteur de mythes qui élude les questions mieux qu'il n'y répond?

Une philosophie prolétarienne de l'histoire consiste à postuler ce miracle que la dictature emploie les armes de la bourgeoisie sans devenir quelque chose comme une bourgeoisie, qu'une classe dirige sans entrer en décadence, quand toute classe qui régit le tout s'avère, par là même, particulière, qu'une formation historique, le prolétariat, puisse être instituée en classe dirigeante sans reprendre les servitudes du rôle historique, qu'elle accumule et garde intacte en elle-même toute l'énergie de toutes les révolutions passées, anime sans défaillance son appareil institutionnel et en annule à mesure la dégénérescence. 33

D'autre part, et ce n'est pas rien, il faut dire combien l'entreprise révolutionnaire contemporaine, si on y est attentif, s'exprime en retrait de la masse et qu'elle "évacue le

29. Aventures, p. 133. Nous soulignons.

30. Aventures, pp. 132 et 307

31. Comme l'a rappelé en juin 1989 , en Chine, la tuerie légalisée des * contre-révolutionnaires* (!), suivie des exécutions publiques et barbares, «le prolétariat comme suppression de soi-meme (Selbstaufhebung), est introuvable. A Aventures, p. 133

32. Aventures, p. 141

33. Aventures, p. 322. 
terrain du prolétariat comme classe dirigeante et laisse en déshérence le mystère de civilisation que, selon Marx, le prolétariat occidental portait. »34

On saisit à la fin que l'égarement marxiste ne doit pas être recherché en dehors de son propre libellé. Il se révèle au travers de la conception du rapport théorie-praxis asphyxiant à la source l'élan critique de ladite théorie. En consommant cette mixture dans laquelle le prolétariat, comme par incantation, devient le messager de vérité par sa fusion au dialectique, le projet de libération se soustrait totalement à la réflexion sur lui-même. Loin de nous offrir un regard alerte, aux aguets du réel aussi bien que de ses propres manquements, ce projet délègue son pouvoir de penser à un muscle social qui, de la sorte, s'improvise comme chemin de la raison. C'est la messianique mécanique du prolétariat-vérité. Doucereusement, la ciguë sommeillait au creuset de

l'idée marxiste d'une critique incarnée historiquement, d'une classe (...) bref, le matérialisme, qui, sous une apparence de modestie, fait de la critique marxiste un dogme et l'empêche d'être une autocritique. ${ }^{35}$

Or si la vérité de la théorie se confond avec un secteur de la société, il en résulte que tout mouvement de celui-ci se voit légitimé d'office. On retrouve alors «chez ses représentants, la conviction d'être l'universel en acte, le droit de s'affirmer sans restriction, la violence invérifiable. „36

Cette idée de violence, virtuellement présente dans la théorie, amène le philosophe à adopter un ton particulièrement sévère à l'endroit des essais récemment publiés de son compagnon de route intellectuel, Jean-Paul Sartre. ${ }^{37}$ Hormis ce qui sépare la pensée de ce dernier de la charte marxiste (sous la tutelle de Staline, à l'époque) - et Sartre marque clairement cette distance 38 - M.-Ponty estime que le "marxisme sartrien" poursuit en droite ligne le suicide théorique opérant déjà depuis de nombreuses années.

Certes, Sartre a horreur du matérialisme sous toutes ses formes. Cependant, il perpétue l'objectivisme qu'il y dé-

34. Aventures, p. 327

35. Aventures, p. 338

36. Idem.

37. Il s'agit de "Les Communistes et la paix* et * Réponse à Claude Lefort *, textes parus dans Les Temps Modernes en 1952-54.

38. Il fait état de son « désaveu de la * matière * historique, de la classe comme mesure de l'action et de la révolution comme vérité. * Sartre fustige en outre le * finalisme honteux qui se cache sous toutes les dialectiques. Aventures, p. 183 et $\mathrm{S}$. cité p. 208. 
nonce en lui offrant un nouveau support fort paradoxal, à savoir le cogito comme parolier du sens - justifiant tout parce qu'il part de rien sinon de lui-même :

Dès que la conscience intervient, elle intervient en législateur souverain, parce que c'est elle qui donne le sens (...) total ou nul. On reconnaît le cogito. C'est lui qui donne à la violence sa nuance sartrienne (...) jusqu'à l'obscurité de l'«action pure. . $^{39}$

Le matérialisme historique entendait se suffire à luimême. De façon analogue, le cogito ne tolère pas la critique : étant la signification même, il ne saurait se récuser sans du coup s'annihiler. Pourtant il faut bien des "raisons" pour être marxiste, et des motifs pour avaliser une action plutôt qu'une autre : il n'est pas inscrit dans le cogito qu'il doive être communiste. C'est ici que Sartre débouche sur un moralisme invétéré du "devoir-être" qui n'a rien à envier aux Fondements de la Métaphysique des mours. Car ce qui lui permet de s'associer au projet marxiste, c'est avant tout la "volonté catégorique de faire être ce qui n'a jamais été. " 40 Pure liberté en effet, le cogito désire ardemment et immédiatement sa propre concrétion. Il devient inutile de s'encrasser dans les subtilités intellectuelles du dialectique et du concept de prolétariat. Plongeons allègrement dans la mythologie de l'Histoire!

Ia liberté je la veux ici et maintenant, semble crier le Je-veux-pensant sartrien. ${ }^{41}$ La difficulté, c'est qu'en voulant se sortir de l'aveugle matière, le philosophe se retrouve vraisemblablement dans un opposé qui demeure tout aussi abstrait. ${ }^{42}$ Cette abstraction prend la forme d'un purisme qui, en politique, se coiffe rapidement du chapeau de l'intolérance, avant de revêtir celui de la violence (il faut se souvenir comment la "Liberté absolue " de l'après Révolution française devint terreur en s'inspirant des Lumières de Rousseau et Kant notamment, hommes on ne peut plus pacifiques...). C'est pourquoi Merleau-P. reprend du même mouvement les deux extrêmes : «La philosophie de l'objet pur et celle du sujet pur sont également terroristes. " ${ }^{43}$

39. Aventures, pp. 232 et 233 . "La lumière pure est l'obscurité pure, * disait bellement Hegel.

40. Aventures, p. 147. Nous soulignons. Transparaît ici l'* extrémisme* propre à l'aventure de Sartre - et peut-être bien, après tout, à son etre psychologique. On soupçonne déjà chez lui, d'autre part, ses raisons non marxiennes de s'avouer * marxiste *.

41. M.-P. utilisera d'ailleurs l'expression d'-universel immediat., empruntée à Hegel, pour caractériser le type d'engagement que privilégie Sartre.

42. L'empirisme et le formalisme, disait encore Hegel, sont des frères jumeaux.

43. Aventures, p. 146. 
Mais pourquoi parler de pureté et la fondre si résolument à la violence? C'est que sous une forme ou l'autre on se croit autosuffisant, et on échappe de la sorte à toute remise en question. À vrai dire, bolchéviques et l' «ultra-bolchévique " ${ }^{44}$ s'entendent pour éviter l'homme concret, l'humain pétri de chair et d'histoire, éprouvé par la vie et par sa propre conscience : ou bien, confiant, on n'a qu'à suivre les croûtons laissés par le Petit Poucet prolétarien, qui nous conduiront infailliblement à la Liberté (la dialectique * fonctionne* toute seule); ${ }^{45}$ ou bien nous essayons de nous reconnaître en ce sujet abstrait qui, par sa minceur existentielle même, peut s'évertuer à se croire tout-puissant. Le cogito (étant volonté aussi bien que raison : « je suis condamné à la liberté ) ) est en effet investi de tous les possibles dans la mesure où, précisément, il s'abstrait abstraitement de son caractère déterminé d'homme plongé dans les servitudes de la quotidienneté. Parallèlement, le double refuge en des lieux éthérés et inaccessibles conditionne le refus de la parole et de l'acte provenant d'un ailleurs. De là, l'opposition-trahison et le dirigisme-vérité qui s'appellent mutuellement dans l'aveuglante lumière. On aboutit forcément alors à des réflexions de ce genre :

(...)Et si le dirigeant se trompe? Comment se tromperait-il? répond Sartre. On peut se tromper de chemin quand le chemin existe. Mais quand il est tout à faire (...) le chemin choisi est le seul possible, et a fortiori le meilleur. ${ }^{66}$

\section{Marx, ou ce qu'il fallait démontrer}

Merleau-Ponty a été séduit par la pensée marxiste. Puis sa réflexion l'amène à prendre ses distances vis-à-vis de ce discours qui, depuis Engels jusqu'à Sartre, révèle un vice profond : la violence du dogmatisme. Perplexe, notre auteur s'interroge : comment une philosophie de la liberté a-t-elle pu devenir l'instrument de la tyrannie?

Il fallait sans doute s'y attendre: "La contradiction et l'équivoque sont (...) dans le réalisme de Marx. Nous disons bien de Marx, et non pas seulement du bolchévisme. " ${ }^{47}$ Les concepts élaborés par le maître ne sont pas toujours claire-

44. C'est le qualificatif que l'auteur des Aventures assène à son compatriote.

45. Mais on constate l' « imprévu * : les oiseaux du conte de Perrault d'une part, les hommes bien réels refusant de passer par le trou de serrure de la dialectique des choses d'autre part.

46. Aventures, Pp. 219 et 220

47. Aventures, p. 127. 
ment distingués de la matière qu'ils sont supposés rendre intelligible. On assiste progressivement chez Marx, affirme le commentateur, surtout à partir de 1848-1850, à l'établissement d'une connexité entre ce que l'on appelait plus haut, avec Weber, le sens et la force. L'idée se mêle ainsi au fait, et celui-ci devient droit. La révolution n'est plus souhaitable, mais inévitable; le prolétariat ne regroupe plus des ouvriers, il incarne le Christ de la religion nouvelle; la dialectique disparaît comme œil vigilant qui saisit et in tègre les contradictions, elle se fait loi implacable des choses.

Le matérialisme dialectique qui se voulait scientifique s'est peu à peu transformé en un matérialisme plus près de l'alchimie que de la science. L'intention de comprendre le réel en vue de le modifier s'est muée en un système philosophico-économique sclérosé, et s'appuyant exclusivement sur sa propre parole. D'où la difficulté constante de départager dans le texte marxien l'instrumentation dialectique, inhérente à la théorie, de ce qui par ailleurs semble valoir comme les lois de son objet. Persiste donc, chez Marx lui-même, ce "conflit de la pensée dialectique et du naturalisme ${ }^{48}$, dont on a beaucoup parlé, qui n'est pas vraiment tranché, et qui laisse en plan le jeu de cache-cache de l'un par l'autre. Cette épine - qui, en dernier ressort, écorcha la chair non moins profondément que la pensée - dispose M.-Ponty à écrire au sujet du "vieux" Marx que

ce qu'il cherche chez Hegel, ce n'est plus l'inspiration dialectique, c'est le rationalisme, pour le faire jouer au bénéfice de la " matière», des « rapports de production », considérés comme un ordre en soi, une puissance extérieure et toute positive (...) il s'agit d'annexer à l'économie la logique de Hegel. ${ }^{49}$

De Marx à Staline et retour. Non, indiscutablement, l'auteur du Capital ne saurait être blanchi du dictatoriat contemporain, qui du reste s'en est réclamé avec tout le prosélytisme qu'on lui connaît ${ }^{50}$

48. Aventures, p. 97.

49. Aventures, p. 95

50. Malgré Raymond Aron, selon lequel * Marx n'est pas pris au sérieux par la majorité des intellectuels soviétiques pour qui le marxisme est simplement une phraséologie *. Opinion exprimée dans le Magazine littéraire de septembre 1983, qui lui a été consacré. Heureusement, et contre toute attente, le paysage soviétique et européen a profondément changé depuis. Gorbatchev sera vraisemblablement le dernier Napoléon du second millénaire. Il n'est pas sans intéret, par ailleurs, de constater plusieurs lieux de rencontre entre Aron et l'auteur de Humanisme et terreur, et ce malgré les divergences étalées parfois assez vertement. Ainsi, dans $L$ 'Opium des intellectuels (publié la méme année que Les Aventures, et où M.-P. est notamment pris à partie), l'auteur considère que le bobo n'est pas chez Staline, mais plutot : *Il y avait une conception du mouvement révolutionnaire qui devait aboutir à ce qui est devenu l'Union soviérique. . 
Dans la perspective, on aperçoit, au bout de ce développement, le marxisme d'aujourd'hui : (...) l'action qui changera le monde, ce n'est plus la praxis philosophique et technique indivises, mouvement des infrastructures, mais aussi appel à toute la critique du sujet, c'est une action du type technicien comme celle de l'ingénieur qui construit un pont. ${ }^{51}$

\section{Merleau-Ponty : la nuance *et * l'ambiguïté}

Ce n'est pas l'effet du hasard si cette pensée, profondément vouée à la liberté peut-être comme nulle autre (par sa présence au concret mais aussi, et malgré tout, par la rigueur qui l'animait), devint le gourdin d'un Staline. En réclamant la liberté et l'égalité pour tous les hommes, on a cru trop aisément que la critique de la lettre signifiait le refus de cette liberté et de cette égalité. Le marxisme contemporain a confondu littéralement la véritable liberté et le chemin qui prétend y mener - l'ceuvre et l'outil. On a troqué l'aliénation économique pour une aliénation idéologique à faire rêver le plus vil des libéralismes. In terroger Marx, Lénine ou Staline est vite apparu insupportable. L'édifiante entreprise intellectuelle, amorcée par le génial penseur allemand, a cessé de se considérer comme un programme de compréhension/transformation de la réalité : elle s'est travestie en religion dont on doit accepter les décrets, sous peine d'excommunication voire même de mort, comme le fidèle se soumet aux dogmes de l'Église. De toute façon, puisque les * choses *, stricto sensu, ne parlent point, il est dès lors vain de les interroger. C'est le culte du matérialisme. Par le sceptre de la dialectique, la théorie autorisait une métaphysique du Prolétariat-Pravda et le roman de la Révolution orgasmique. C'est la voie qui fut suivie.

Reste à savoir si une autre version était possible. Certes, Merleau-P. renvoie le marxisme à Marx, mais sa pensée se montre à cet égard particulièrement flottante. Ainsi, ses critiques à l'endroit de Marx sont-elles peu fréquentes et dispersées ici et là dans l'analyse où elles perdent de leur mordant, même dans Les Aventures. Le ton et la formulation trahissent également les velléités du propos. En langage psychanalytique, on dirait que l'auteur ne se résout pas à "abattre " le Père. Serait-ce qu'il n'est pas convaincu de ses propres conclusions, ou bien se refuse-t-il à croire que Marx ait véritablement sinon entraîné, du moins suscité le drame 
actuel ?52 Plus tard notamment, en 1960, il tiendra un discours qui ne va pas sans rappeler Humanisme et terreur: "Ne confondons pas le marxisme et le communisme. " 53 De celui-là, dit-il encore, on «peut garder une sérieuse valeur heuristique. " ${ }^{54}$ Or on aimerait bien examiner les raisons qui amènent le philosophe à lui conserver cet honneur, alors que le ver ronge déjà de l'intérieur l'œuvre originale. Bref, le texte du maître peut-il offrir une alternative au matérialisme dogmatisant ? Soit en accordant une plus large faveur à certains travaux (ceux de jeunesse, spécialement), soit en considérant cet objectivisme comme une interprétation qui mériterait d'être révisée à la lumière d'autres lectures possibles. Sinon, il faudra se contenter de récupérer quelque objet précieux du domicile en flamme, et ainsi dénaturer la pensée en l'utilisant hors de ses cadres épistémologiques.

Il semble que le penseur français soit tenté par cette dernière éventualité. Sous cet angle, on comprendrait en tout cas que le marxisme «n'est certainement plus vrai dans le sens où il se croyait vrai " 55 . Mais peut-on alors persister en toute légitimité à s'entretenir de "marxisme"? En d'autres termes, Marx accepterait-il qu'on aborde son ouvre à la manière d'une philosophie, et qu'on y cueille les fruits selon les humeurs et les modes? Il est permis d'en douter : à la façon de l'hégélianisme, le marxisme est un système tissé dans une logique qui lui est propre. Quoi qu'il en soit, on demeure dans l'incertitude quant à l'interprétation à donner au texte marxien. Irrésolu, M.-Ponty reste donc dans l'ambiguité.

52. Il ne prend pas d'ailleurs une position précise sur l'œuvre de Marx. Il est symptomatique de le voir relever (voir supra, p. 108-110) l'* equivoque $*$, le * conflit * et la * contradiction * du texte sans pousser la recherche qui permettrait de mieux cerner la difficulté. En se contentant de mettre le doigr sur le problème sans remonter à ses conditions, et en n'en examinant pas de façon serrée le dénouement dans l'étroit couloir qui relie Marx aux disciples (a cet égard on saute trop rapidement, croyons-nous, de Marx aux marxistes), l'auteur perpétue l'équivoque qu'il dénonce. Aussi, en laissant Marx dans l'ambivalence, la sienne se revèle comme d'autant plus convenable. Nous retenons l'hypothèse que si l'auteur ne s'engage pas plus avant dans ce contexte, ce n'est pas tellement par respect des nuances que parce que l'essentiel de l'analyse reste encore à faire. Si le germe bafouille déjà chez Marx - et que celui-ci annonce l'endémie du totalitarisme moderne sous les couleurs de la liberté - il importe en effet de le soumettre avec empressement au plus fameux de nos microscopes. Mais, sans nier l'importance de la question, M.-P. nous aurait peut-etre signalé que ses intentions écaient modestes, et surtout plus ancrées dans la terre glaise du moment. Nous le voyons bien nous dire que Marx sera encore sur sa table de travail demain, que c'est aujourd'hui que Staline baillonne en Sibérie et conduit ses chars tantôt à Berlin, tantốt à Budapest. Cela dit, le problème n'est pas simple.

53. Signes, p. 14. On remarquera par surcroît que c'est le marxisme - et non seulement Marx - qu'il tient ici à distinguer de la politique totalitariste

54. Signes, p. 15

55. Signes, p. 15 
Un certain malaise, d'autre part, accompagna notre lecture. Comme on l'a vu, l'auteur fait abondamment référence à plusieurs théoriciens marxistes. Or il est étrange de constater qu'il y est très peu fait mention de leur pensée-dans-letexte, et que la citation laisse place le plus souvent à la paraphrase. Semblable démarche, à notre avis, exige du lecteur une confiance qu'en toute rigueur il ne peut offrir. Une construction élaborée de la sorte risque fort, au contraire, de faire naître chez celui-ci une prudence peutêtre excessive, voire même la méfiance. Nous ne sommes pas convaincu par ailleurs de la fermeté de l'argumentation qui s'inspire de certains travaux pour, semble-t-il, en éloigner d'autres de valeur comparable sinon supérieure. On voudrait parfois comprendre pourquoi l'auteur retient des documents et des thèses aux dépens de quelques autres demeurés ignorés. L'importance d'Empirisme et Empiriocriticisme, pour ne citer qu'un exemple, ne fait pas de doute; on sait qu'elle fut son influence. Toutefois, on se serait attendu à être informé plus justement de la singularité de cet ouvrage eu égard à l'ensemble de la pensée de Lénine, au demeurant plus riche que ne le laisse en trevoir ce texte. 56 Dans le même ordre d'idées, il est permis de questionner les motifs qui amènent à conserver le silence sur les Gramsci, Luxembourg, Korsch et autres grands personnages du marxisme. Est-ce de manière purement fortuite qu'on a fait intervenir dans la discussion des ouvrages et des penseurs qui, finalement, corroborent les thèses avancées? Avouons qu'il eût été difficile de dénoncer l'objectivisme marxiste par le biais d'un Korsch. ${ }^{57} \mathrm{Si}$ les absents ont toujours tort, encore faut-il avoir la courtoisie de les inviter au dialogue.

Pourtant, nous partageons l'essentiel des vues de M.-Ponty. Quelque peu informé d'un Sartre moins « intransigeant", d'un Lénine plus "souple " et d'un Trotsky moins "abstrait", la critique du philosophe nous est apparue tout de même très juste. Nous ajouterions de surcroît qu'il n'est pas certain que le dernier tiers de siècle nous ait offert ("Nouveaux philosophes" inclus) un commentaire réellement plus pénétrant des véritables failles du système. ${ }^{58}$

56. Il faut dire en revanche que M.-P. fit quelques courts commentaires à ce sujet, entre autres à l'égard de Que faire?, et par l'intermédiaire de Korsch au surplus. Mais ça reste très mince.

57. Il est vrai que Lukacs prend une importance non négligeable dans l'analyse; mais n'est-ce pas dans un jeu d'ombres pour mieux degager le positivisme léninien, qui d'ailleurs obligea ce premier à rentrer dans le rang.

58. Si on excepte peut-etre la réflexion de Raymond Aron dont on supportait, à l'époque, d'autant moins l'intelligence qu'il l'appliquait avec force pour dénoncer le marxisme contemporain. Il n'est pas facile d'avoir raison avant tout le monde! Sartre est lumineux aussi dans ses Questions de métbode, nonobstant la «violence* maintenue dans la Critique de la raison dialectique. 
Nous eûmes préféré cependant qu'il nous eût convaincu de la pertinence de son propos sans recours à ce favoritisme indu dans le choix des textes.

\section{Merleau-Ponty, philosophe}

Il est bon de fréquenter des esprits libres qui cherchent honnêtement des issues aux problèmes sans chercher du même coup à se complaire, comme réfugié en quelque tour de Babel, dans les solutions désignées. Merleau-Ponty est de ceux-là. Ils ne sont pas sans savoir que nous vivons dans le provisoire, et que *l'assurance d'être porteur du vrai est vertigineuse. Elle est pour elle-même violence. "59 Sur papier ou en discussion entre amis, tout un chacun applaudira à de telles paroles. De quelle façon cependant y donnonsnous suite dans nos activités quotidiennes? La violence est un Protée aux mille figures subtiles, combien plus faciles à identifier lorsqu'on en est la victime que lorsqu'on en est le levier. Machiavel ne rappelle-t-il pas en outre que «le comble de la tyrannie est de séduire "? ${ }^{60}$ De la séduction-amour à la séduction perfide - de la douceur à la douleur - la distance est parfois insensible.

C'est peut-être avant tout en cette prise de conscience que réside le rôle fondamental du philosophe, à savoir : renvoyer toute prise de position à ses conditions de possibilité, la soumettre à des lumières nouvelles sous le feu de l'interrogation perpétuelle. Car toute proposition, dans son caractère déterminé, recèle sa dose d'a-rationalité. Littéralement, agir signifie cesser de réflécbir, mettre fin aux délibérations sur l'action à entreprendre. Tel est tout à la fois la beauté et la rançon de la finitude. ${ }^{61}$ Or ce n'est certes pas dans la remise en question constante, protestera-t-on avec raison, qu'on fera pousser le blé dans les contrées ou défendra notre langue et notre culture devant l'envahisseur. Pourtant, si le concept d' "action raisonnable» n'est pas totalement dénué de sens, il faut bien que celle-ci sache recevoir les hommages de la critique. C'est dire qu'elle doit obtempérer à la mise en relation avec autre-chose-qu'ellemême, et ce pour se voir com-prise dans son rapport au

59. Aventures, p. 190

60. Aventures, p. 302

61. *L'instant de la décision est une folie, * dit Kierkegaard. Parce que * toute action est manichénne, ajoute Malraux. On comprendra la formule de M.-Ponty : * Les manicheens qui se heurtent dans l'action s'entendent mieux entre eux qu'avec le philosophe. Eloge p. 69 
monde. Bref, n'est-ce pas privilégier un objet que le penser : on ne réfléchit qu'à ce qui déjà, au départ, revêt une valeur possible, sinon probable. ${ }^{62}$ L'objet ne peut que ressortir grandi de son voyage dans les eaux érosives mais purifiantes de la raison. Grandi, ou détruit! On ne se libère véritablement de son esclavage que dans le risque de la mort, disait encore Hegel. Mieux vaut certainement dissoudre l'idée pâteuse ou fausse dans l'arène philosophique, plutôt que la laisser mordre la chair des individus dans la réalité bien concrète de la vie des hommes.

Refuser le crible de la raison, c'est refuser de dévoiler le caché, le non-dit. C'est donc favoriser l'erreur et en quelque façon la bêtise, sans compter l'ignorance. Mais voilà! il n'est pas aisé de s'y soumettre. Pareil défi exige beaucoup d'humilité et de courage. On comprendra pourquoi Socrate demeure au fond l'ultime référence de la réflexion philosophique. Tout en étant un esprit fort et pénétrant - ce qui lui eût permis de dominer et donc de violenter ses pairs -, celui-ci a compris, grâce à cet esprit même, qu'«il ne savait rien.» Qu'est-ce à dire sinon que la sagesse éclot du sol rocailleux de la pensée rigoureuse, par laquelle nous apprenons incidemment qu'il n'y a point de lieu de vérité échappant à un questionnement ultérieur.

À nos yeux le texte de M.-Ponty, et ce malgré les réserves que nous formulions plus haut, renvoie au cheminement socratique parce que s'y manifeste précisément cette union de l'implacable dialectique du penser - qui cherche non pas à justifier mais à comprendre - avec l'humilité de celui qui sait que les résultats de sa réflexion demeurent fragiles. Pas étonnant qu'il ait dénoncé avec grande sagacité une pensée déjà violente dans sa phrase en se posant comme dogme irréfutable. Contrairement à un grand nombre d'intellectuels, surtout de ces années orageuses, notre auteur échappa à cet engouement de l'anti ou du pro-marxisme. Et il serait erroné d'apercevoir en cela une conduite fade et complaisante (on doit rappeler son enthousiasme d'après-

62. Comme l'a magistralement montré Hegel, penser/comprendre consiste précisément dans le rapport à la négativité, selon laquelle la chose se definit dans son autre : omnis determinatio est negatio, disait Spinoza. Sur le plan etymologique, on sait en outre que intelligence provient de intelligere, qui signifie comprendre, lequel se décompose à nouveau en prendre-avec. On remarquera par ailleurs que *penser* tire son origine du meme vocable latin, pensare (réfléchir), qui donna naissance à *panser *. Nous insistons sur l'approche hégélienne dans notre ouvrage sur La Rationalité vivante. Essai sur la pensée hégélienne, paru à Québec au printemps de 1990, aux Éditions Le Griffon d'argile. Nous insistons tout particulièrement sur ce caractère de relation du penser dans la section terminale du livre, portant sur "Aimer, penser, mourir *, pp. 133-152. 
guerre, par exemple, qui le disposa à donner son assentiment même aux Procès de Moscou). Bien sûr, cette *attitude du comprendre" prête le flanc au regard oblique de celui qui prend position, disons, plus nettement. ${ }^{63}$ Mais en dernier ressort, est-ce le rôle véritable du philosophe que de se déterminer de la sorte? Ne sont-ils pas légion ceux qui se fixent en des idées et des programmes et, parmi le nombre, à élever le ton et à défendre leur opinion avec virulence jusqu'à prendre les armes le cas échéant, pour ne pas permettre à quelques-uns de simplement « voir » plus clair afin de mieux présenter les données du problème ?64 Pour théorique qu'elle soit, la philosophie n'en est pas moins une praxis. Le philosophe n'a pas d'idole. Il ne possède que sa raison qu'il cherche à ne pas prostituer dans la fermeture (éclair!) d'une idée qu'on estime définitive. Ou plutôt si, cette idole se nomme Liberté : celle de ne rien considérer comme limite absolue à son pouvoir d'interroger et de remettre à la question, bref la liberté de penser. Ceci implique - et c'est peut-être l'essentiel - qu'il s'éprouve luimême comme insuffisant. Il n'échappe pas, en effet, à la douloureuse négation qu'il s'inflige par le regard corrosif de sa propre raison qui le harcèle. Aussi, aperçoit-il la nécessité de voyager par et dans ses entours. Comme le souligne magnifiquement M.-Ponty, le philosophe a profondément besoin de "différence" : "Notre rapport avec le vrai passe par les autres. " 65

Entendons qu'il ne s'agit nullement de faire l'apologie du philosophe qui détiendrait, par la raison et la liberté, les rênes d'un monde meilleur. A prôner la raison et la liberté - et leurs corrélatifs que sont l'écoute et la tolérance - le philosophe n'en est pas quitte pour autant à considérer

63. * Meme s'il n'a jamais trahi [le philosophe], on sent, à sa manière d'être fidèle, qu'il pourrait trahir, il ne prend pas part comme les autres. Eloge, p. 69.

64. Cela signifie-t-il un retrait somme toute calcule sur les coussins de sa chaise de travail? Lorsque M.-P. annonce son adouble parti pris de poser le problème social en termes de lutte et de refuser la dictature du prolétariat (...) la résolution de garder en main les deux bouts de la chaine, le problème social et la liberté,* il ajoute: «Ceci n'est pas « une solution * et nous le savons bien.* Aventures, pp 330, 331 et 332. Pas plus que quiconque, il ne connaît la recette d'une société où chacun est heureux, riche et en santé. Il a le mérite cependant de montrer l'erreur, qui est celle de considérer unilatéralement les deux «maillons* (en l'occurence, le liberalisme et le communisme, respectivement planqués en leurs tranchées). Le philosophe travaille à élaborer une pensée nuancée sans laquelle l'action ne saurait etre éclairée. Il aurait fait sienne la position de Bergson : - Il faut agir en homme de pensée et penser en homme d'action.- Opportunisme, compromis, réconciliation tiède et nauséabonde? Non point. Ne pas confondre modestie et résignation ou, pis encore, avec une collaboration à l'exploitation de fait.

65. Eloge, p. 45 . 
ces/ses fleurons du discours à la manière d'un nouvel impératif catégorique (ne serait-ce pas à nouveau bafouer la liberté, en effet, que de la présenter dans les habits kaki du commandement?). Tout au contraire, il s'agit de montrer qu'une réflexion rigoureuse mène - nous dirions presque nécessairement -à la prise de conscience de notre extrême déficience. Par conséquent, l'Autre me devient beaucoup plus salutaire comme compagnon qu'adversaire dans une lutte de pouvoirs. ${ }^{66}$ - $\mathrm{A}$ quel XXème siècle aurions-nous eu droit si cette pensée imbue de liberté qu'est le marxisme avait compris qu'elle commettait son suicide en refusant la liberté d'être... pensée?

"Les révolutions sont vraies comme mouvements et fausses comme régimes. " écrit notre auteur avec justesse. ${ }^{67}$ Il est à se demander si ce n'est pas la liberté inhérente à la raison (entraînée par delà elle-même selon la dynamique de son dedans) ${ }^{68}$ qui peut le plus profondément rencontrer cette exigence de ne point s'asseoir sur soi. L'idée n'a rien d'étrange si, effectivement, «le sens de la révolution est d'être révolutionnaire, c'est-à-dire critique universelle et en particulier critique d'elle-même." 69

Lac-Beauport, P.Q.

66. On retrouve le cercle de la pensée - qui refuse les décrets, se nommeraient-ils liberté et raison. La raison aspire à découvrir ses bornes et ses paramètres dans le labeur de son propre questionnement. Une fois de plus, on rejoint Hegel par une idée terrible de profondeur: *C'est la réflexion qui élève le vrai a un résultat. „ Celui-ci l'opposera incidemment à la manière dogmatique de penser, à savoir * l'opinion selon laquelle le vrai consiste en une proposition qui est un résultat fixe ou encore en une proposition qui est immédiatement sue. Phénoménologie de l'Esprit (1807), Paris, Aubier-Montaigne, 1939, pp. 19 et 35.

67. Aventures, p. 303.

68. Et c'est là le principal thème sur lequel se penche notre ouvrage déjà cité.

69. Aventures, p. 84. 
Bibliographie des travaux à portée politique de Maurice Merleau-Ponty

1947 - Humanisme et terreur. Essai sur le problème communiste. (Humanisme) Recueil d'articles publiés en 1946 et 1947 , et en volume en 1947. Avec une introduction de Claude Lefort en 1980. Paris, Gallimard, Idées, 1980.

1948 - Sens et Non-Sens. (Sens) Recueil d'articles publiés de 1945 à 1947, en volume en 1948. Paris, Nagel, 1966. À noter que la pagination de cette édition diffère de l'originale.

1953 - Éloge de la Pbilosophie et autres essais. (Éloge) L'Éloge fut le texte de la leçon inaugurale donnée au Collège de France, le 15 janvier 1953. Les autres essais furent précédemment publiés sous forme d'articles depuis 1947 à 1960, année où les textes furent rassemblés en volume. Paris, Gallimard, Idées, 1968.

1955 - Les Aventures de la dialectique. (Aventures) Paris, Gallimard, Idées, 1977.

1960 - Signes. (Signes) Recueil d'articles et de textes de conférence écrits ou publiés de 1947 à 1960. Paris, Gallimard, NRF, 1960. 